\title{
Catastrophic antiphospholipid syndrome: A case report and literature review
}

\author{
Luis del Carpio-Orantes ${ }^{1}$, Chantall Citlally Anaya- Martínez ${ }^{1}$ and Elías Bonilla -Casas ${ }^{2}$ \\ ${ }^{1}$ Intensive Care Unit; '2Department of General Surgery Hospital General de Zona No. 71, Delegación Veracruz Norte, Instituto Mexicano del Seguro \\ Social, Veracruz, Ver., Mexico
}

\begin{abstract}
The present document is the report of a case of a very rare clinical entity, which presents with acute multiorganic failure after a thrombotic storm related to antiphospholipid antibodies, the so-called catastrophic antiphospholipid syndrome (CAPS), which began as a recurrent picture of mesenteric thrombosis, with a previous history of venous insufficiency and distal ulcers probably associated with an unidentified antiphospholipid; deserving management in intensive care and the consultation by the world expert, Dr. Ricard Cervera who confirmed the diagnosis and recommend treating as such entity, the patient's evolution was satisfactory so far. Final recommendations for diagnosis and current treatment options such as rituximab or eculizumab are made. The present case was added to the international registry that currently houses around 500 cases worldwide (International CAPS Registry).
\end{abstract}

KEY WORDS: Catastrophic antiphospholipid syndrome. Mesenteric thrombosis. Multiorgan failure.

\section{Case Presentation}

The case is presented of a 52-year-old male with a history of peripheral vascular ulcers and chronic postphlebitic syndrome (Fig. 1), who was admitted with symptoms of acute abdominal pain in addition to ileus, pneumatosis intestinalis, and free fluid evidenced by abdominal tomography (Figs. 2-4). He underwent exploratory laparotomy, with the findings of intestinal necrosis, omental necrosis, and 1800-cc hemoperitoneum, which were considered to be secondary to segmental mesenteric thrombosis, with small bowel resection $(80 \mathrm{~cm})$, omentectomy, and ileostomy being performed. Symptoms recurred, which deserved a second surgical intervention, with the patient developing multiorgan failure (renal, pulmonary, hepatic, hematologic [disseminated intravascular coagulation], cardiovascular, and probably adrenal) in $<7$ days. This time, he underwent distal ileum resection $(60 \mathrm{~cm})$ due to ischemia and ileostomy. He was admitted to the intensive care unit where he was provided hemodynamical and mechanical ventilation support. He experienced a third abdominal event with stomal necrosis that required remodeling.

In view of the above, a thrombophilia protocol was initiated given the recurrent intestinal thrombotic condition, which was mainly characterized by the presence of anticardiolipin immunoglobulin $\mathrm{G}(\mathrm{IgG})$ and IgM antibodies with weak positivity, together with histopathological evidence of coagulative necrosis and transmural bowel hemorrhage, as well as omental necrosis and vascular congestion. Given our doubts about the case, we asked Dr. Ricard Cervera, world expert in this pathology, for his assessment, after which he kindly suggested that this could be a case of catastrophic antiphospholipid syndrome (CAPS) and recommended treating it as such, using low molecular weight heparin (enoxaparin, $1 \mathrm{mg} / \mathrm{kg}$ per day) and methylprednisolone pulses ( $3 \mathrm{~g} /$ day), with

\section{Correspondence:}




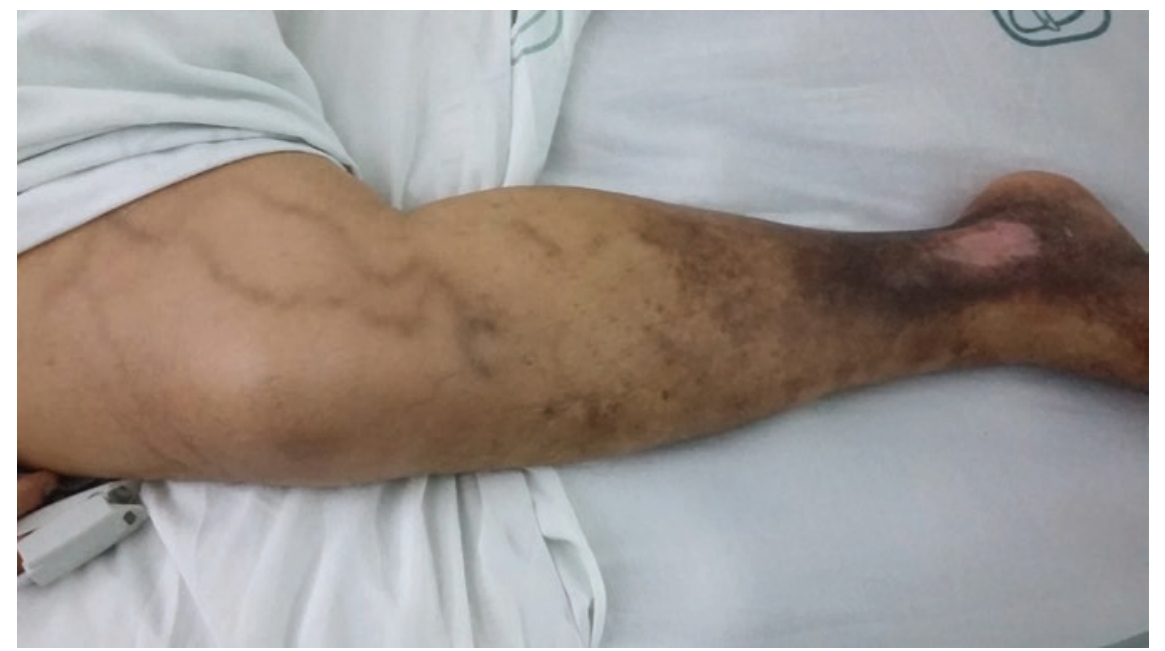

Figure 1. Chronic postphlebitic syndrome and distal cicatricial ulcer as manifestations of not previously identified antiphospholipid syndrome.

subsequent maintenance doses and intravenous $\lg G$ $(0.4 \mathrm{~g} / \mathrm{kg} /$ day for 5 days).

Evolution was satisfactory, with remission of all organ lesions, except for the liver lesions, the remission of which was slow and gradual. The patient is currently stable at the intensive care unit, already without mechanical ventilation support or amines, and has some healthcare-associated infections (pneumonia and surgical wound infection) in progress of being controlled, as well as critical illness myopathy. He has undergone entero-entero anastomosis due to previous ileostomy, with good tolerance (Table 1).

The patient was included in the International CAPS Registry. We consider that ulcers and chronic postphlebitic syndrome can be a manifestation of a previously undetected primary APS and that it is correlated with the catastrophic condition the patient presented with.

\section{Discussion}

This case meets all criteria to be considered catastrophic APS, with acute intestinal ischemia as an acute event that derived in recurrent necrosis together with multiorgan failure, most likely due to intestinal thrombosis and target organs (kidneys, liver, adrenal glands, and lungs), microthrombosis, with histopathological examination demonstrating data consistent with coagulative necrosis, edema, and vascular congestion, as well as transmural hemorrhage. During the study of thrombosis for suspected concomitant thrombophilia, slightly elevated antiphospholipid antibodies were reported, which was corroborated with a second determination together with the elevation of other antibodies, related in this case to extractable nuclear antibodies (ENA), with anti-SSB or anti-La elevation being reported, which is an unusual association in CAPS. We considered that chronic lesions in the patient's lower limbs, as well as ulcer-related scars and postphlebitic syndrome, may be related to a previously undetected primary APS that became evident with this catastrophic presentation. Given our doubts, due to the rareness of this diagnosis, we consulted with the world expert, who contemplated the CAPS diagnosis and recommended treating the case as such disease, which drove us to choose an unfractionated heparin, systemic steroids, and intravenous Ig treatment scheme, with the good evolution of the patient.

\section{Review}

CAPS, also called Asherson syndrome, has its first descriptions in 1984, but it was in 1992 when Asherson (1934-2008) described it as a generalized coagulopathy that is strongly related to antiphospholipid antibodies, but completely different and separate of other inherited or acquired coagulopathies ${ }^{1,2}$. This definition has evolved, and currently, it is considered to be a thrombotic storm with microvascular and macrovascular occlusions in a short period of time, with poor prognosis in spite of receiving treatment.

The incidence of CAPS is $1 \%$ of all APS cases, and it is regarded as a rare, but lethal, variant of this syndrome. In the International CAPS Registry last review, 500 cases, recorded between 1992 and 2004, are referred, with new cases being continuously added to the registry such as the present one ${ }^{3}$. As regards diagnosis, diagnostic criteria have been established for defined or confirmed catastrophic APS, namely: 


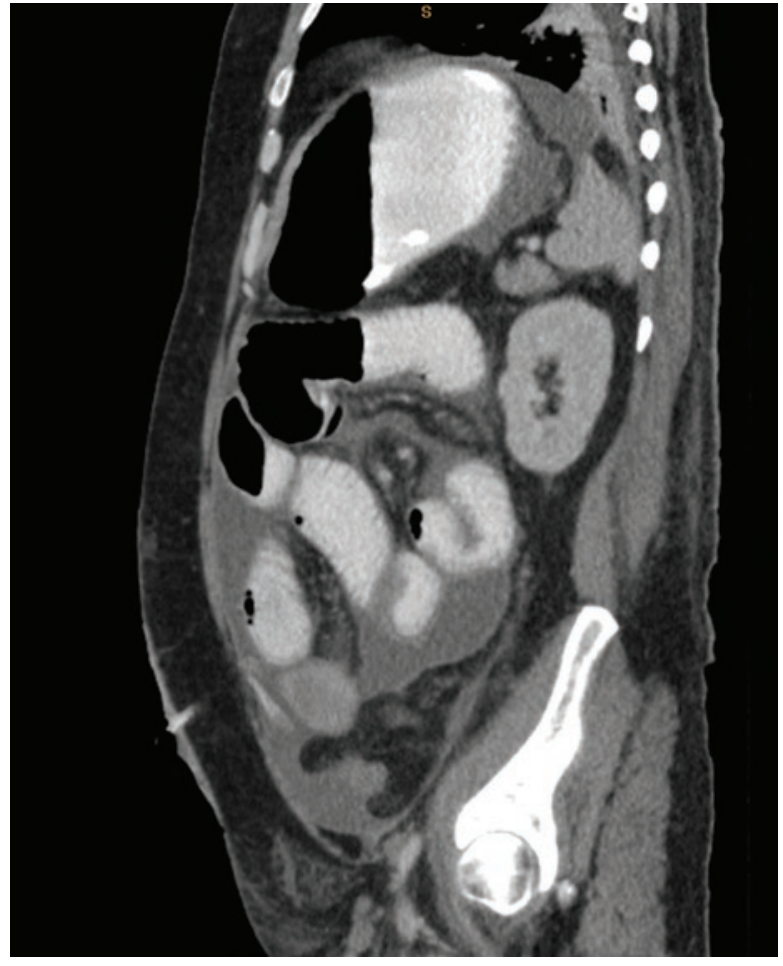

Figure 2. Data consistent with ileus, free abdominal fluid, and pneumatosis intestinalis.

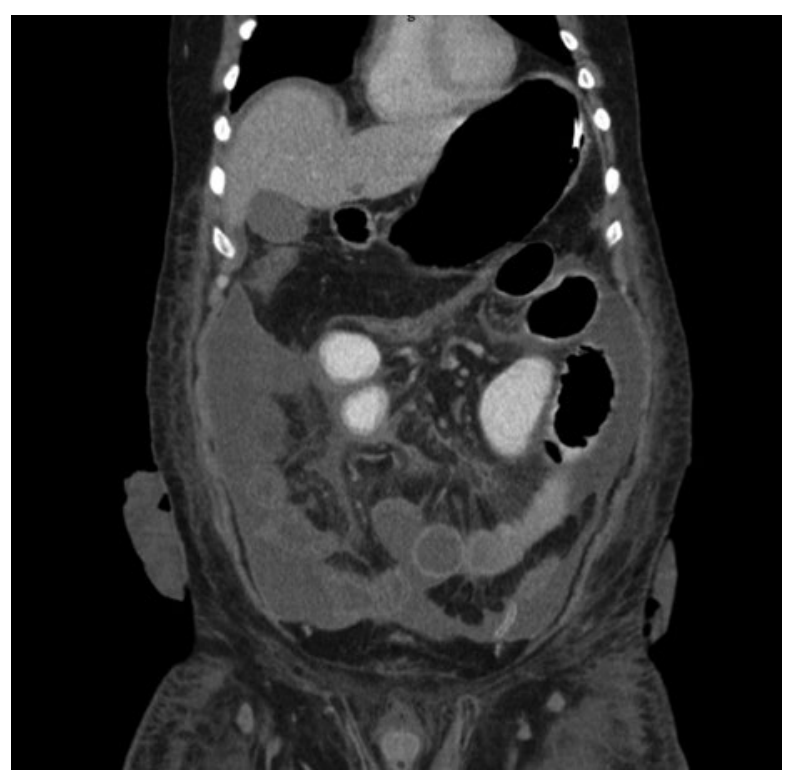

Figure 3. Data consistent with ileus and pneumatosis intestinalis, as well as with gastric chamber distension.

1. Involvement of three or more organs, systems, or tissues.

2. The presence of multiorgan lesion in $<7$ days.

3. Histopathological confirmation of small vessel occlusion.

4. The presence of antiphospholipid antibodies.

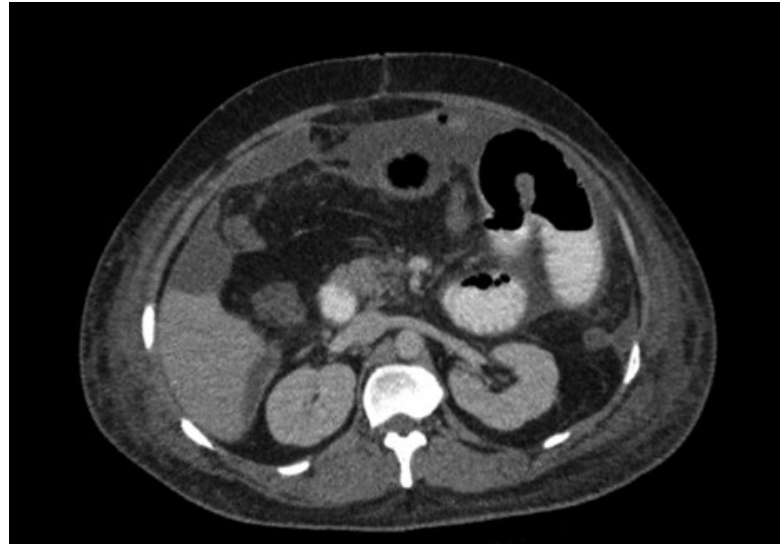

Figure 4. Ileus and pneumatosis intestinalis as manifestations of mesenteric thrombosis.

Probable APS is when not all the above criteria are met, but it can include combinations thereof that make for the likelihood of the syndrome to increase:

- All four criteria, but involvement of only two organs, systems, or tissues.

- All four criteria, except for the presence of antiphospholipid antibodies.

- Criteria 1, 2, and 4.

- Criteria 1, 3, and 4, but with the development of a third event at between 8 and 30 days of the first one, in spite of anticoagulation ${ }^{4-8}$.

Currently, in the International CAPS Registry last report (2016), mortality is observed to have decreased from $50 \%$ (in initial reports) down to $33 \%$, probably owing to higher identification of the condition at early stages and opportune treatment, although systemic lupus erythematosus-associated CAPS cases are reported to have the highest mortality rate, with $48 \%$. Most affected gender continues to be the female gender $(69 \%)$, and most affected age group is the $31-39$ year group, with an average of 38 years of age. $60 \%$ of cases have corresponded to primary APS, and 30\% have been associated with systemic lupus erythematosus. Among the predominant factors, infections $(49 \%)$, surgery $(17 \%)$, and malignancy $(16 \%)$ stand out ${ }^{3}$.

Organ failure most common manifestations are renal $(73 \%)$, pulmonary $(60 \%)$, neurological $(56 \%)$, cardiac $(50 \%)$, and cutaneous (47\%). Most relevant biochemical findings are thrombocytopenia (67\%) and the presence of lupus anticoagulant (83\%), anticardiolipin IgG antibodies (81\%), and anti-B2 glycoprotein IgG antibodies (78\%). Antinuclear antibodies are present only in $57 \%$, anti-DNA in $32 \%$, and anti-ENA in $10 \%{ }^{3}$. 
Table 1. Biochemical characteristics of the patient at hospital admission, during the critical stage (multiorgan failure) and at current stage

\begin{tabular}{|c|c|c|c|}
\hline & Admission & Critical stage (5-7 days) & Current stage (50 days) \\
\hline $\mathrm{Hb} / \mathrm{WBC}$ & $\begin{array}{c}11.3 \mathrm{~g} / 14,900 \\
\mathrm{~mm}^{3}\end{array}$ & $6.3 \mathrm{~g} / 33,600 \mathrm{~mm}^{3}$ & $8.9 \mathrm{~g} / 9000 \mathrm{~mm}^{3}$ \\
\hline Platelets & 206,000 & 10,000 & 117,000 \\
\hline Glucose & $55 \mathrm{mg}$ & $100 \mathrm{mg}$ & $69 \mathrm{mg}$ \\
\hline Urea/creatinine & $92 / 1.6 \mathrm{mg}$ & $135 / 3.5 \mathrm{mg}$ & 18/0.6 mg \\
\hline $\mathrm{Na} / \mathrm{K}^{+}$ & $149 / 3.9 \mathrm{mmoL}$ & $128 / 4.5 \mathrm{mmoL}$ & $138 / 4.3 \mathrm{mmoL}$ \\
\hline Total bilirubin/DB & $5.2 / 4.5 \mathrm{mg}$ & $14 / 10.2 \mathrm{mg}$ & $9 / 7.6 \mathrm{mg}$ \\
\hline AST/ALT & $75 / 85 \mathrm{U}$ & $165 / 148 U$ & $48 / 56 U$ \\
\hline LDH & $399 \mathrm{U}$ & $679 \mathrm{U}$ & $330 \mathrm{U}$ \\
\hline PT/PTT & $23.7 / 24.3 \mathrm{~s}$ & $21.8 / 61 \mathrm{~s}$ & $12.8 / 38 \mathrm{~s}$ \\
\hline Fibrinogen & - & $167 \mathrm{mg}$ & $440 \mathrm{mg}$ \\
\hline D-dimer & - & $4,800 \mu \mathrm{g}$ & - \\
\hline Coombs & - & Negative & - \\
\hline Schistocytes & - & Negative & - \\
\hline Antinuclear antibodies & - & 0.56 index (negative) & 0.41 index (negative) \\
\hline Lupus anticoagulant & - & Negative & Negative \\
\hline Anticardiolipin IgG & - & 11.46 (weak positive) & 18.2 U/GPL (positive) \\
\hline Anticardiolipin IgM & - & 10.06 (weak positive) & $<2$ U/MPL \\
\hline Anti-DNA & - & - & 17.39 IU (negative) \\
\hline Anti-Sm & - & - & 0.31 ratio (negative) \\
\hline $\mathrm{C} 3 / \mathrm{C} 4 / \mathrm{CH} 50$ & - & - & CH50 $95.8 \cup$ (low) \\
\hline Anti-B2 glycoprotein & - & - & $6.0 \mathrm{U} / \mathrm{mL}$ \\
\hline Anti-SSA (Ro) & & & 0.71 ratio (negative) \\
\hline Anti-SSB (La) & & & 1.57 ratio (positive) \\
\hline Clinical status & $\begin{array}{l}\text { Acute abdomen } \\
\text { postphlebitic } \\
\text { syndrome }\end{array}$ & $\begin{array}{l}\text { Multiorgan failure, shock, disseminated } \\
\text { intravascular coagulation, hypoxemia, } \\
\text { generalized jaundice, anasarca, encephalopathy }\end{array}$ & $\begin{array}{l}\text { Jaundice in remission, critical } \\
\text { illness myopathy, postphlebitic } \\
\text { syndrome }\end{array}$ \\
\hline
\end{tabular}

As regards treatment, single-drug and combined treatments have been examined. Among single-drug treatments, the most widely used are anticoagulants $(82 \%)$, steroids $(76 \%)$, plasmapheresis $(35 \%)$, and intravenous Ig $(27 \%)$. Among the combined treatments (higher effectiveness, in theory), anticoagulants with steroids $(19 \%)$ and anticoagulants + steroids + plasmapheresis/intravenous $\lg (18 \%)$ stand out ${ }^{9-11}$.

Recently, monoclonal antibodies have been used for the treatment of CAPS, but evidence is still scarce and there are only anecdotal case reports. Rituximab was used in 33 patients (6\% of the series) and eculizumab in 2 patients $(0.2 \% \text { of the series })^{12}$. Two cases have been reported where renal graft thrombosis prevention was performed in patients with CAPS with good results; however, conducting trials with larger numbers of patients are recommended. There is one case report where eculizumab was used in a female patient who had received steroids and cyclophosphamide, with renal damage persisting and requiring hemodialysis, but following the use of the antibody, discontinuing such therapy was possible due to an important improvement of the renal function ${ }^{13}$. 
Similarly, another case report of a male subject demonstrated an improvement that had not been accomplished with anticoagulation, immunosuppression, and plasma exchange. In conclusion, eculizumab might be preemptively implemented in patients with CAPS that are to undergo renal transplantation and in those in whom conventional therapies have failed to control the disease, as well as in those with recurrent symptoms. According to conclusions drawn from the International CAPS Registry, rituximab is recommended for patients with CAPS that is refractory to conventional treatment ${ }^{11-15}$

As treatment summary, all patients with CAPS should be treated with anticoagulants, steroids, and plasmapheresis/intravenous Ig. All patients with CAPS associated with systemic lupus erythematosus should be additionally treated with cyclophosphamide (according to current evidence, patients without lupus may worsen if they are administered cyclophosphamide), and for refractory cases or relapses, the use of monoclonal antibodies such as rituximab or eculizum$\mathrm{ab}$ is fully recommended.

Referred factors of poor prognosis include younger age (mainly in the 31-39-years age group), belonging to the female gender and association with systemic lupus erythematosus, which confers a higher mortality rate.

\section{Acknowledgments}

The authors would like to thank Dr. Ricard Cervera Segura, Senior Consultant Physician and Head of the Department of Autoimmune Diseases, Hospital Clinic of Barcelona, Head of the Research Team on Systemic Autoimmune Diseases at the Institut
d'Investigacions Biomèdiques August Pi i Sunyer (IDIBAPS), Barcelona, and Professor at the Department of Medicine, University of Barcelona, Spain, where he coordinates the Masters Course on Autoimmune Diseases, who always was willing to clarify our doubts and to direct us for the resolution of the present case.

\section{References}

1. Asherson RA. The catastrophic antiphospholipid syndrome. J Rheumatol. 1992;19:508-12.

2. Sciascia S, López-Pedrera C, Roccatello D, et al. Catastrophic antiphospholipid syndrome (CAPS). Best Pract Res Clin Rheumatol. 201s2;26:535-41.

3. Rodríguez-Pintó I, Moitinho M, Santacreu I, et al. Catastrophic antiphospholipid syndrome (CAPS): Descriptive analysis of 500 patients from the International CAPS Registry. Autoimmun Rev. 2016;15:1120-4.

4. Cervera R, Rodríguez-Pintó I, Colafrancesco S, et al. $14^{\text {th }}$ International Congress on Antiphospholipid Antibodies Task Force Report on Catastrophic Antiphospholipid Syndrome. Autoimmun Rev. 2014;13:699-707.

5. Cervera R. CAPS Registry. Lupus. 2012;21:755-7.

6. Asherson RA, Cervera R, Piette JC, et al. Catastrophic antiphospholipid syndrome. Clinical and laboratory features of 50 patients. Medicine (Balt). 1998;77:195-207.

7. Asherson RA, Cervera R, Piette JC, et al. Catastrophic antiphospholipid syndrome: Clues to the pathogenesis from a series of 80 patients. Medicine (Balt). 2001;80:355-77.

8. Aguiar CL, Erkan D. Catastrophic antiphospholipid syndrome: How to diagnose a rare but highly fatal disease. Ther Adv Musculoskelet Dis. 2013;5:305-14.

9. Cervera R, Rodríguez-Pinto I, Espinosa G. Catastrophic antiphospholipid syndrome: Task force report summary. Lupus. 2014;23:1283-5.

10. Kazzaz NM, McCune WJ, Knight JS. Treatment of catastrophic antiphospholipid syndrome. Curr Opin Rheumatol. 2016;28:218-27.

11. Tenti S, Cheleschi S, Guidelli GM, et al. Intravenous immunoglobulins and antiphospholipid syndrome: How, when and why? A review of the literature. Autoimmun Rev. 2016;15:226-35.

12. Berman H, Rodríguez-Pintó I, Cervera R, et al. Rituximab use in the catastrophic antiphospholipid syndrome: Descriptive analysis of the CAPS registry patients receiving rituximab. Autoimmun Rev. 2013;12:1085-90.

13. Strakhan M, Hurtado-Sbordoni M, Galeas N, et al. 36-year-old female with catastrophic antiphospholipid syndrome treated with eculizumab: A case report and review of literature. Case Rep Hematol. 2014;2014:704371.

14. Shapira I, Andrade D, Allen SL, et al. Induction of sustained remission in recurrent catastrophic antiphospholipid syndrome via inhibition of terminal complement with eculizumab. Arthritis Rheum. 2012;64:2719-23.

15. Lonze BB, Zachary AA, Magro CM, et al. Eculizumab prevents recurrent antiphospholipid antibody syndrome and enables successful renal transplantation. Am J Transplant. 2014;14:459-65. 